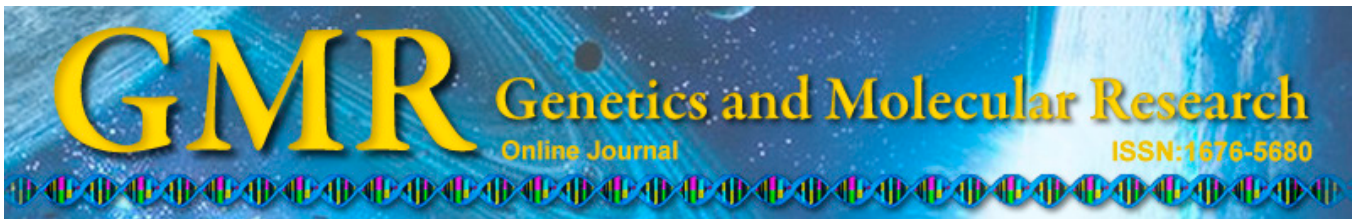

\title{
Preliminary study of the effects of $\beta$-elemene on MCF-7/ADM breast cancer stem cells
}

\author{
Y. Dong, L. Li, L. Wang, T. Zhou, J.W. Liu and Y.J. Gao \\ First Affiliated Hospital of Medical University, Dalian, China \\ Corresponding author: Y.J. Gao \\ E-mail: gaoyajie_gyj@yeah.net
}

Genet. Mol. Res. 14 (1): 2347-2355 (2015)

Received May 19, 2014

Accepted October 20, 2014

Published March 27, 2015

DOI http://dx.doi.org/10.4238/2015.March.27.20

\begin{abstract}
We examined expression differences in breast cancer stem cells (BCSCs) of the doxorubicin-resistant breast cancer cell line MCF-7/ADM and doxorubicin-sensitive cell line MCF-7/S. The effects of Chinese medicine $\beta$-elemene on BCSCs and resistance protein expression were determined. The serum-free cell culture method was used for cell culture, and morphology was observed to determine the rate of cell sphere formation. Reverse transcription-polymerase chain reaction was used to detect breast cancer resistance protein $(B C R P)$ and P-glycoprotein $(P-g p)$ gene expression. Flow cytometry was used to determine BCRP- and P-gp-positive cell rates and CD44 + CD24-/low cell ratios. Morphological observation and gene amplification showed that compared with MCF-7/S cells, the serum-free cell sphere-forming rate and $P$ - $g p$ and $B C R P$ gene expression levels were higher in MCF7/ADM cells. Flow cytometry results showed that P-gp and BCRP protein expression in MCF-7/ADM cells was $77.78 \pm 9.55 \%$ and 32.33 $\pm 5.12 \%$, respectively, and the CD44 + CD24-/low cell rate was 64.79 $\pm 11.78 \%$, which were all significantly higher than those in MCF-7/S cells $(3.97 \pm 1.51,14.26 \pm 2.51,18.79 \pm 3.28 \% ; \mathrm{P}<0.05)$. $\beta$-elemene significantly decreased the serum-free cell sphere-forming rate in MCF7/ADM cells and BCRP and $\mathrm{P}$-gp gene/protein expression $(\mathrm{P}<0.01)$.
\end{abstract}


The proportion of CD44 + CD24-/low cells was reduced. MCF-7/ADM highly expressed the drug-resistant proteins BCRP and P-gp, which can be used for long-term in vitro culture and as a seed cell for studies of BCSCs. $\beta$-elemene can inhibit BCSC and the sphere-forming rate in $\mathrm{MCF}-7 / \mathrm{ADM}$ cells and reduce BCRP expression.

Key words: Breast cancer stem cells (BCSCs); Drug resistance protein; $\beta$-elemene

\section{INTRODUCTION}

Recently developed cancer stem cells have been used to increase the understanding of the biological characteristics of tumor cells, such as tumor occurrence, development, metastasis, and drug resistance. They have also been used in the development of treatments for malignant tumors. Al-Hajj et al. (2003) successfully isolated, cultured, and identified human breast cancer stem cells (BCSCs), which were the first stem cells to be isolated in solid tumors. This discovery has raised new possibilities for breast cancer treatment. Elemene is a second-class broad-spectrum anticancer drug in China; the main component is $\beta$-elemene ( $\beta$-ELE), and its anti-tumor effects are mainly achieved via high concentrations to directly kill tumor cells, induce apoptosis and differentiation, and inhibit blood vessel growth (Zhou et al., 2000; Tang et al., 2010). However, few studies have examined the impact of $\beta$-ELE on BCSCs. Because BCSCs cannot be stably maintained for long periods of time using in vitro culture methods, we used MCF-7/ADM BCSCs to study the effects of $\beta$-ELE on BCSCs. These cells contain high levels of $\beta$-ELE and show similar biological characteristics to BCSCs.

\section{MATERIAL AND METHODS}

\section{Cell culture and experimental grouping}

The human breast cancer cell line MCF-7/S and doxorubicin-resistant cell line MCF7/ADM were purchased from KGI Biotechnology Development Co., Ltd. (Nanjing, China). MCF-7/S cells were routinely cultured in RPMI 1640 medium containing 10\% fetal bovine serum, $200 \mathrm{U} / \mathrm{L}$ insulin, $100 \mathrm{U} / \mathrm{mL}$ penicillin, and $100 \mu \mathrm{g} / \mathrm{mL}$ streptomycin. MCF-7/ADM cells were cultured in RPMI 1640 medium containing $10 \%$ fetal bovine serum, $1000 \mathrm{ng} / \mathrm{mL}$ ADM, $100 \mathrm{U} / \mathrm{mL}$ penicillin, and $100 \mu \mathrm{g} / \mathrm{mL}$ streptomycin (ADM was added to maintain the resistance of cells). Cells were placed at $37^{\circ} \mathrm{C}, 5 \% \mathrm{CO}_{2}$ in a $95 \%$ humidity incubator for long-term subculture. Two weeks before the experiment, the MCF-7/ADM cells were placed in culture medium without doxorubicin. All experimental cells were used in the logarithmic growth phase. Experimental cells were divided into 3 groups: MCF-7/S group, MCF-7/ADM group, and low-cytotoxic $\beta$-ELE MCF-7/ADM group.

\section{Reagents and instruments}

$\beta$-ELE was purchased from Jingang Pharmaceutical Co., Ltd. (Dalian, China). Ethylenediaminetetraacetic acid (EDTA) was from Amvesco Co. (Eugene, OR, USA). Trypsin was from Sigma (St. Louis, MO, USA). Dimethyl sulfoxide was from Life scientific instru- 
ment company. B27 was from PAA Company (Piscataway, NJ, USA). Doxorubicin was from Zhejiang Hisun Pharmaceutical Co., Ltd. (Shanghai, China). Insulin was from Wanbang Biochemical Pharmaceutical Co., Ltd. (Jiangsu, China). RPMI 1640 medium, Dulbecco'u modified Eagle medium (DMEM)/F12, fetal bovine serum, and 2 antibodies were purchased from Gibco (Grand Island, NY, USA). Basic fibroblast growth factor (bFGF) and epidermal growth factor (EGF) were purchased from PeproTech, Inc. (Rocky Hill, NJ, USA). RNAiso Plus Total RNA extraction reagent, PrimeScript II First-Strand cDNA Synthesis Kit, and TaKaRa Taq enzyme kit were purchased from Takara Bio-Engineering Co., Ltd. (Shiga, Japan). The cell culture incubator was from SANYO Co., Ltd. (Osaka, Japan). Clean Bench was from Shanghai Boxun Co. (Shanghai, China). The instrument used for polymerase chain reaction (PCR) was from Hangzhou Jingle Scientific Instrument Co. (Hangzhou, China). The inverted microscope was from Leica (Wetzler, Germany). The flow cytometer was from BD Biosciences (Franklin Lakes, NJ, USA). The ultraviolet (UV) spectrophotometer was from Thermo Scientific (Waltham, MA, USA).

\section{Serum-free cell culture methods}

Cells in the logarithm growth phase were pipetted and digested to prepare a single-cell suspension. Phosphate-buffered saline (PBS) was used to wash the cells twice, followed by trypan blue staining and counting. Serum-free DMEM/F12 containing growth factors (1:50 B27, $20 \mathrm{ng} / \mathrm{mL}$ EGF, $20 \mathrm{ng} / \mathrm{mL}$ bFGF, $1 \%$ anti-Bis) was used to resuspend the cells. Cells were inoculated at $2 \times 10^{6} / \mathrm{mL}$ on a $3.5-\mathrm{cm}$ culture dish and then placed in an incubator at $37^{\circ} \mathrm{C}, 5 \% \mathrm{CO}_{2}$, $95 \%$ humidity. Every other day, $1 \mathrm{~mL}$ serum-free medium (SFM) was added to the culture dish. The growth of CMSs was observed under a microscope each day. The mammosphere suspension (MSs) formed in approximately 14 days. After MS formation, passaging was performed approximately once every 5 days; pipetting was performed gently until most MSs had disappeared. The samples were centrifuged at $1000 \mathrm{rpm}$ for $5 \mathrm{~min}$. The supernatant was discarded and MSs were collected. These cells were further cultured in SFM as described above.

\section{Induced differentiation of cancer stem cell spheres after serum-free culture}

Cell spheres in suspension growth under serum-free culture were pipetted gently until most MSs had disappeared. The sample was centrifuged and the supernatant was discarded. After washing twice with PBS, cells were cultured in RPMI 1640 medium containing 10\% serum. Induction of differentiation and growth of cancer stem cells were observed under a microscope.

\section{Reverse transcription (RT)-PCR detection}

For total cellular RNA extraction, the RNAiso Plus kit was used according to manufacturer instructions. UV spectrophotometry was used to detect the purity and concentration of RNA. For cDNA synthesis, RT was conducted according to the instructions of the PrimeScript II First-Strand cDNA Synthesis Kit. For PCR amplification, upstream and downstream primers of mRNA in each gene were used, including: MDR1 5'-GCTCCTGACTATGCCAAAGC-3' and 5'-TCTTCACCTCCAGGCTCAGT-3'; the amplified fragment length was $202 \mathrm{bp}$. BCRP 5'-TGCCCAGGACTCAATGCAACAG-3' and 5'-GACTGAAGGGCTACTAACC-3'; the amplified fragment length was 562 bp. GAPDH 5'-ACCCAGAAGACTGTGGATGG-3' and 
5'-TTCTAGACGGCAGGTCAGGT-3'; the amplified fragment length was $201 \mathrm{bp}$. All primers were synthesized by Jiuh Bao Biotechnology (Dalian) Co., Ltd. (Tainan, Taiwan). The following cycle parameters were used: $94^{\circ} \mathrm{C}$ denaturation for $3 \mathrm{~min} ; 94^{\circ} \mathrm{C}$ for $30 \mathrm{~s}, 55^{\circ} \mathrm{C}$ for $30 \mathrm{~s}, 72^{\circ} \mathrm{C}$ for $60 \mathrm{~s}, 30$ cycles; $72^{\circ} \mathrm{C}$ extension for $10 \mathrm{~min}$. PCR products were analyzed on $2 \%$ agarose gels for electrophoresis and ethidium bromide was used for staining. A gel imaging system was used to take photos under UV light. Each RNA sample was subjected to RT-PCR analysis 3 times.

\section{Flow cytometry}

To prepare the single-cell suspension, cells in the logarithmic growth phase were acquired, and the culture medium in the bottle was aspirated and discarded. PBS was used to wash the cells twice to eliminate dead cells. Next, $0.25 \%$ trypsin- $0.02 \%$ EDTA was used to digest cells until the cell cytoplasm retracted under the microscope, the gap increased, after which the process was immediately terminated. A single-cell suspension was prepared by gentle pipetting. After centrifugation, the supernatants were washed twice with PBS to remove the serum and cells were resuspended in PBS. After trypan blue staining and counting, the cell concentration was adjusted to $1 \times 10^{7} / \mathrm{mL}$, with each tube cell containing $5 \times 10^{6}$ cells. Digestion was performed carefully to avoid the effect of excessive digestion on cell viability. Both experimental tubes and control tubes were used. For CD44 and CD24 expression detection, CD44-PE and CD24-fluorescein isothiocyanate antibodies were added to the cells but not to the control tube. After incubation at room temperature for $30 \mathrm{~min}$, a small amount of PBS was added; after centrifugation, the supernatant was discarded and $0.5 \mathrm{~mL}$ PBS was used to resuspend the cells.

The cells were detected using flow cytometry. For P-gp and BCRP expression detection, P-gp-APC or BCRP-PerCP/Cy5.5 antibodies were added to the experimental tubes but not the control tube. After $30 \mathrm{~min}$ incubation at room temperature, a small amount of PBS was added; after centrifugation, the supernatant was discarded and $0.5 \mathrm{~mL}$ PBS was used to resuspend the cells. The cells were detected using flow cytometry.

\section{RESULTS}

\section{Serum-free cell sphere-forming rate}

Three days after the serum-free culture of cells, the cell morphology gradually changed to a triangle shape. The cell gap became larger and the cells formed a network. At approximately 10 days, mammospheres began to form and cell apoptosis in large areas was observed. At approximately 14 days, serum free-cultured mammospheres formed. Figure 1 shows the microscope images of serum-free cell culture on day 14. The sphereforming rate of MCF-7/S cells was low in SFM. The sphere-forming rate of MCF-7/ADM cells in SFM was significantly higher than that of MCF-7/S cells. When $\beta$-ELE acted on MCF-7/ADM cells in SFM, the sphere-forming rate was lower than that of MCF-7/ADM cells. There were significant differences in the content of BCSCs among the 3 groups (Figure 1). 

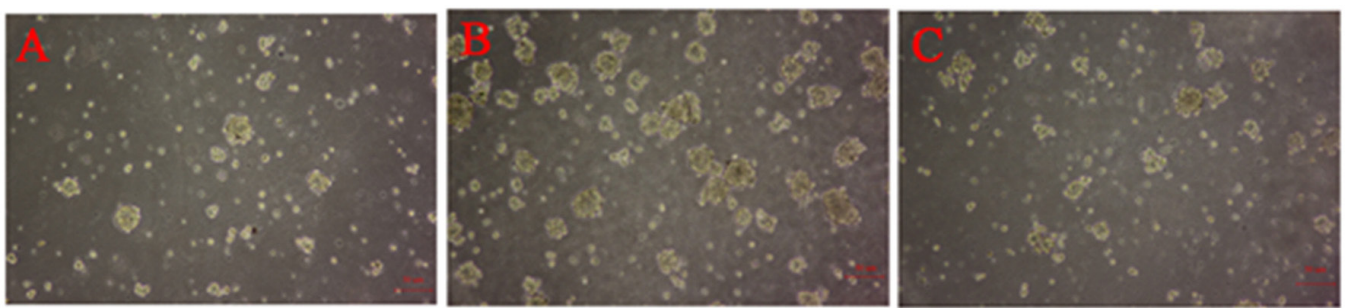

Figure 1. Serum-free cell sphere formatting rate under inverted microscope. A. MCF-7/S cells; B. MCF-7/ADM cells; C. Cell sphere-forming rate of $\beta$-elemene-treated MCF-7/ADM cells. The scale bar is $50 \mu \mathrm{M}$.

\section{Serum free-cultured and sphere-induced differentiation of cancer stem cells}

Serum free-cultured spheres were placed back into common medium containing serum and allowed to adhere to the plate. An inverted microscope was used to observe cell morphology. There was no significant difference in original cell morphology and drug resistance.

\section{$P$-gp and BCRP gene transcription levels using RT-PCR detection}

P-gp and BCRP are both resistant proteins that belong to the ATP-binding cassette (ABC) superfamily. We used RT-PCR to detect the $P$-gp and $B C R P$ gene transcription levels in MCF-7/S cells, MCF-7/ADM cells, and $\beta$-ELE-treated MCF-7/ADM cells. The results are shown in Figure 2. $P$ - $g p$ and $B C R P$ gene transcription levels in MCF-7/ADM cells were significantly higher than those in MCF-7/S cells. After adding $\beta$-ELE, the transcription levels of both genes significantly decreased (Figure 2).

a

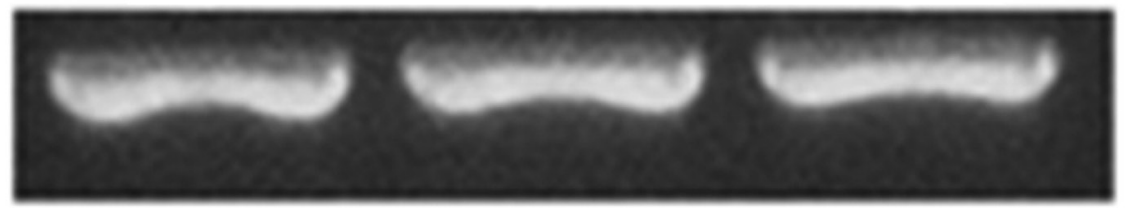

GAPDH

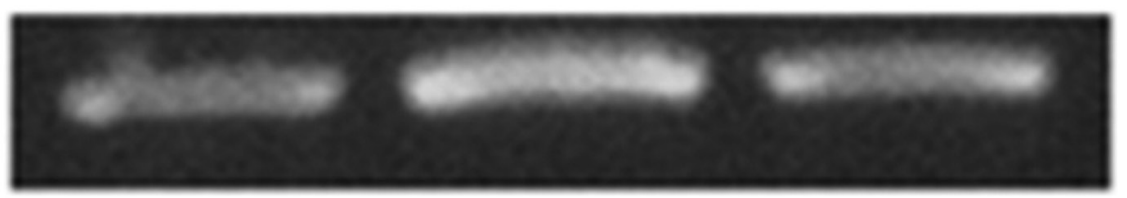

P-gp

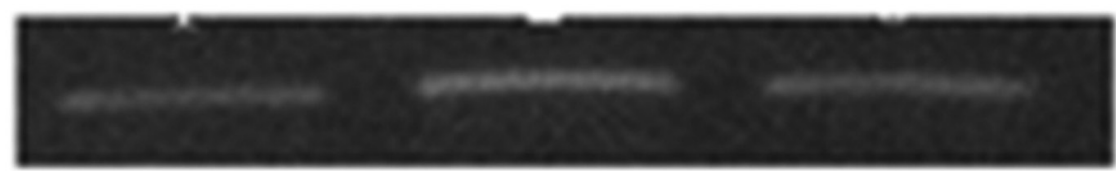

Figure 2. $P$-g $p$ and $B C R P$ gene expression in different cells using RT-PCR detection. Lane $a=\mathrm{MCF}-7 / \mathrm{S}$ cells; lane $b=$ MCF-7/ADM cells; lane $c=\beta$-elemene-treated MCF-7/ADM cells. 


\section{P-gp and BCRP expression using flow cytometry}

Flow cytometry was used to detect the expression levels of P-gp and BCRP proteins. As shown in Figure 3, P-gp expression in MCF-7/S cells was $3.97 \pm 1.51 \%$, BRCP expression was $14.26 \pm 2.51 \%$; P-gp expression in MCF-7/ADM cells was $77.78 \pm 9.55 \%$ and $\mathrm{BRCP}$ expression was $32.33 \pm 5.12 \%$. Expression of both proteins in MCF-7/ADM cells was significantly higher than the expression levels in MCF-7/S cells $(\mathrm{P}<0.01)$. When $\beta$-ELE acted on MCF-7/ADM cells, P-gp expression was $58.13 \pm 5.69 \%$ and BRCP expression rate was 8.60 $\pm 3.34 \%$, which were both significantly lower than the expression levels in MCF-7/ADM cells $(\mathrm{P}<0.01)$ (Figure 3).
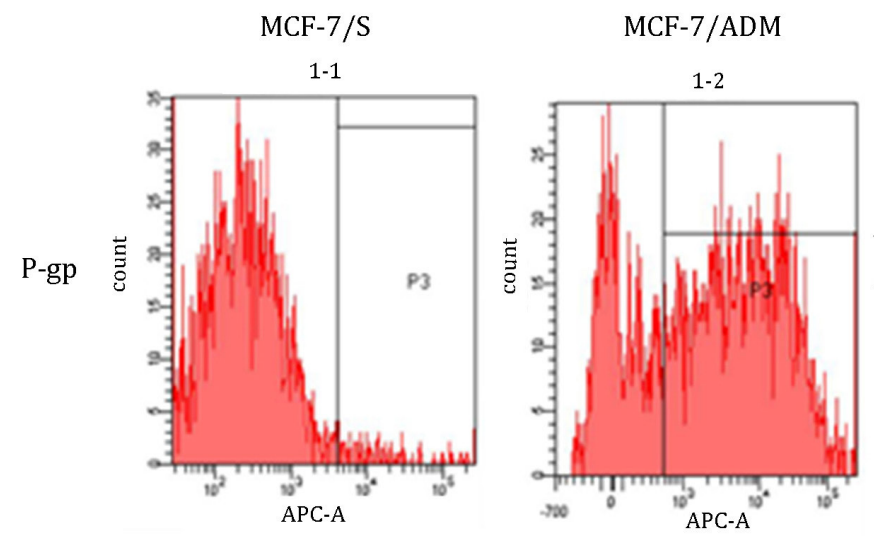

$\beta$-elemene MCF-7/ADM
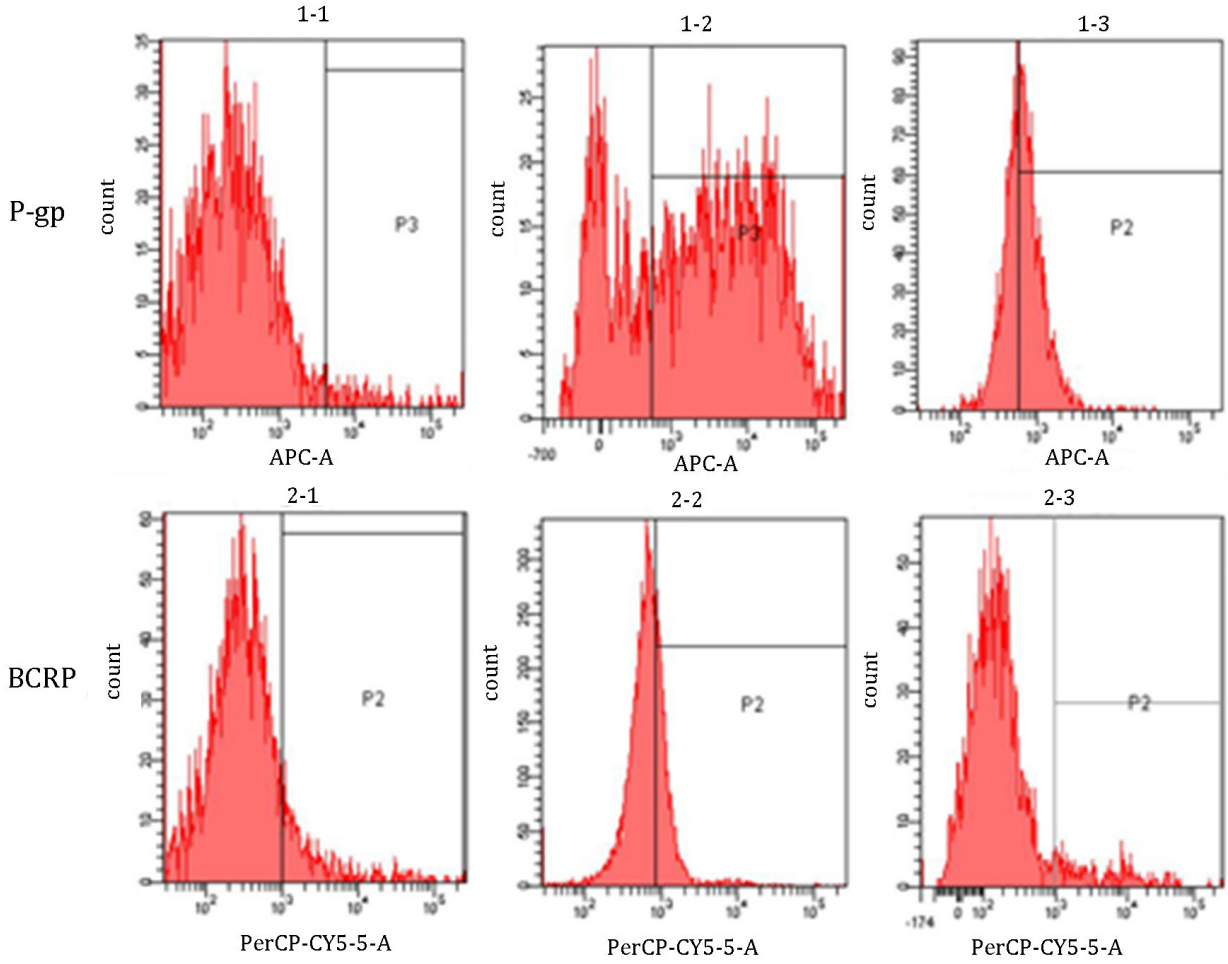

Figure 3. P-gp and BCRP protein expression of different cells using flow cytometry 2.5 CD44 + CD24-/low cell proportion.

CD44 + CD24-/low are the most common surface molecular markers on BCSCs. As shown in Figure 4, in MCF-7/S cells, the proportion of CD44 + CD24-/low cells was $18.79 \pm$ $3.28 \%$. In MCF-7/ADM cells, the proportion of CD44 + CD24-/low cells was $64.79 \pm 11.78 \%$, which was significantly higher than that in MCF-7/S cells $(\mathrm{P}<0.01)$. In $\beta$-ELE-treated MCF7/ADM cells, the proportion of CD44 + CD24-/low cells was $47.15 \pm 3.27 \%$, which was lower than that in MCF-7/ADM cells $(\mathrm{P}=0.067)$ (Figure 4$)$. 

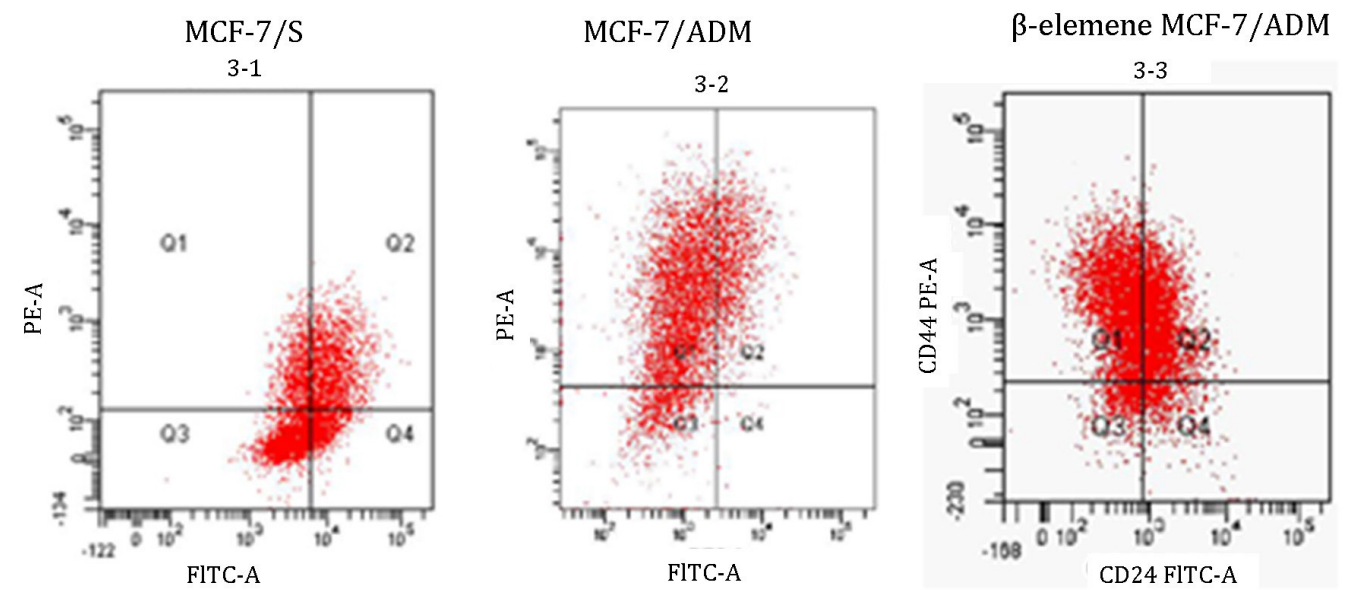

Figure 4. Proportion of CD44 + CD24-/low cells in different cells using flow cytometry.

\section{DISCUSSION}

For in vitro BCSC isolation and identification methods, in addition to sorting based on cell surface molecule markers such as CD44 + CD24-/low 1, a more commonly used method is sorting based on the biological characteristics of cancer stem cells. There are 2 main experimental techniques used for this purpose: side population (SP) method (ABCG2/BCRP method). Kondo et al. (2004) isolated SP cells for the first time in MCF-7 and other tumor cell lines and confirmed that SP cells could differentiate into multiple cell types and had little tumorigenesis activity. They expressed stem cell-like genes showing resistance to chemotherapeutic drugs. High expression of BCRP is considered to be the main factor in generating the SP phenotype (Scharenberg et al., 2002; Clayton et al., 2004). BCRP can maintain the undifferentiated state of stem cells and affect their functions (Zhou et al., 2002). Because the Hoechst3342 staining used in the SP method has toxic effects, the applications of this method are limited. Currently, BCRP has become a candidate marker for isolating and identifying BCSCs (Zhou et al., 2001, 2002). Ponti et al. (2005) cultured neural stem cells with the growth factors DMEM/F12 added and grew cell suspension cultures of primary breast cancer cells and the breast cancer cell line MCF-7. Apoptosis of mature breast cancer cells occurred in this medium. Some of the cells proliferated but gradually formed spherical cell clusters referred to as MSs. MSs were rich in CD44 + CD24-/low cells, did not express differentiation markers, and tumorigenicity significantly increased (Ponti et al., 2005; Phillips et al., 2006). SFM can enrich BCSCs, but MSs cannot be passaged an unlimited number of times in SFM, suggesting that serum-free suspension culture cannot sustain in vitro BCSC amplification for a long period of time.

The $\mathrm{ABC}$ transporter is one of the largest families of transmembrane proteins in the body and functions in the membrane transport of various active substances. The P-gp and $\mathrm{BCRP}$ proteins are multidrug resistance proteins examined in this study. P-gp glycoprotein was the first discovered transmembrane protein to mediate drug transport in the cell; P-gp is encoded by the $A B C B 1$ gene. P-gp has a drug pump function and can detect the drug concentration within cells. When chemotherapy drugs (lipophobic drugs) reach cells and bind to 
P-gp, the drug cytotoxicity may begin to have an effect before it is transported to the extracellular environment. This can decrease the intracellular concentration of drugs; thus, the amount of drug acting on the target nucleus is relatively low, and multidrug resistance would develop more readily. This mechanism of resistance is considered to be a classic resistance mechanism. $\mathrm{BCRP}$ is encoded by the $A B C G 2$ gene; it is the only half transporter protein in drug transportation. The protein has ATP-dependent drug efflux function and is located in the cell membrane. Kawabata et al. (2001) showed that BCRP participates in the formation of active transporter complexes and is necessary for mediation of drug resistance. Through its active transport function, chemotherapy drugs are pumped out of the cells, protecting cells from foreign drug toxicity and directly participating in the tolerance to chemotherapeutic drugs. This is a hotspot for cancer drug research in recent years and is also one of the hotspots in stem cell studies.

In this study, PCR was used to detect the $P$ - $g p$ and $B C R P$ mRNA transcript levels in MCF-7/ADM and MCF-7 cells. We found that $P$ - $g p$ and BCRP expression levels of MCF-7/ ADM were significantly higher than those in MCF-7 cells. Flow cytometry showed that the CD44 + CD24-/low cell rates, BCRP+ cell rates, and P-gp+ cell rates were significantly different between the cell lines. The sphere-forming rate of the 2 types of cells was significantly different in SFM, suggesting that MCF-7/ADM contained more of the BCSC-enriched cell line than did MCF-7. MCF-7/ADM resistance mechanisms may be associated with high expression of P-gp and BCRP proteins. In this study, $\beta$-ELE, which shows low cytotoxicity, was used to treat MCF-7/ADM to detect its effects on the high proportion of BCSCs in the cell line. The PCR, flow cytometry, and serum-free cell sphere culture results showed that the level of mRNA transcription and expression of $P$-g $p$ and $B C R P$ genes were significantly reduced. The CD44 + CD24-/low cell rate and BCRP + cell rate and serum-free cell sphere-forming rate were all decreased.

Only one heterogeneous cell line can be used to express the cell surface markers CD44 + CD24-/low, which shows no direct association with the biological characteristics of BCSCs. CD44 + CD24-/low cells of MCF-7 cells can be affected by culture time, cell density, and culture environment. Whether the fixed molecular phenotype can be used as a marker of cancer stem cells remain controversial (Clarke et al., 2006). BCRP expression rate, serum-free cell sphere-forming rate, and other stable markers of cell surface molecules showed stronger, more direct associations with BCSCs. Therefore, although the results indicated that low-cytotoxicity $\beta$-ELE acted on MCF-7/ADM cells, the CD44 + CD24-/low cell rate decreased but there was no significant difference. However, $\beta$-ELE may have inhibited BCSCs in MCF-7/ ADM cells to some degree. In addition, our experiments showed that lower than cytotoxic doses of $\beta$-ELE can downregulate the transcription and expression of the genes encoding P-gp and BCRP. This indicates that $\beta$-ELE can reverse the multidrug resistance of BCSCs and restore their sensitivity to chemotherapeutic drugs by downregulating the $\mathrm{ABC}$ transporter protein family.

In summary, $\beta$-ELE can relieve the multidrug resistance and progression mechanisms in breast cancer patients with repeated recurrence. Our results provide a theoretical and experimental basis for future studies. Therefore, $\beta$-ELE may play a role in controlling tumor metastasis, recurrence, and drug resistance in cancer stem cells. However, because the methods for isolating, identifying, and detecting proteins are limited to BCSCs in vitro, additional studies are needed to explore the signaling pathways and molecular mechanisms of $\beta$-ELE on the inhibition of BCSCs. 


\section{REFERENCES}

Al-Hajj M, Wicha MS, Benito-Hernandez A, Morrison SJ, et al. (2003). Prospective identification of tumorigenic breast cancer cells. Proc. Natl. Acad. Sci. U. S. A. 10: 3983-3988.

Clarke MF, Dick JE, Dirks PB, Eaves CJ, et al. (2006). Cancer stem cells - perspectives on current status and future directions: AACR Workshop on cancer stem cells. Cancer Res. 66: 9339-9344.

Clayton H, Titley I and Vivanco Md (2004). Growth and differentiation of progenitor/stem cells derived from the human mammary gland. Exp. Cell. Res. 15: 444-460.

Kondo T, Setoguchi T and Taga T (2004). Persistence of a small subpopulation of cancer stem-like cells in the C6 glioma cell line. Proc. Natl. Acad. Sci. U. S. A. 20: 781-786.

Kawabata S, Oka M, Shiozawa K, Tsukamoto K, et al. (2001). Breast cancer resistance protein directly confers SN-38 resistance of lung cancer cells. Biochem. Biophys. Res. Commun. 280: 1216-1223.

Ponti D, Costa A, Zaffaroni N, Pratesi G, et al. (2005). Isolation and in vitro propagation of tumorigenic breast cancer cells with stem/progenitor cell properties. Cancer Res. 65: 5506-5511.

Phillips TM, McBride WH and Pajonk F (2006). The response of CD24(-/low)/CD44+ breast cancer-initiating cells to radiation. J. Natl. Cancer Inst. 98: 1777-1785.

Scharenberg CW, Harkey MA and Torok-Storb B (2002). The ABCG2 transporter is an efficient Hoechst 33342 efflux pump and is preferentially expressed by immature human hematopoietic progenitors. Blood 15: 507-512.

Tang XH, Qin SD and Xie K (2010). Status and progress of basic researches of elemene injection for antitumor. Lin Chuang Zhang Liu Za Zhi 3: 266-273.

Zhou S, Schuetz JD, Bunting KD, Colapietro AM, et al. (2001). The ABC transporter Bcrp1/ABCG2 is expressed in a wide variety of stem cells and is a molecular determinant of the side-population phenotype. Nat. Med. 7: 1028-1034.

Zhou S, Morris JJ, Barnes Y, Lan L, et al. (2002). Bcrp1 gene expression is required for normal numbers of side population stem cells in mice, and confers relative protection to mitoxantrone in hematopoietic cells in vivo. Proc. Natl. Acad. Sci. U. S. A. 17: 12339-12344. 